\title{
ASCA X-Ray Observations of the Vela Pulsar Jet
}

\author{
Hakki Ögelman and Craig Markwardt \\ University of Wisconsin, Madison
}

\begin{abstract}
An X-ray jet-like outflow, beginning at the Vela pulsar and extending about seven parsecs along the pulsar spin axis direction has recently been discovered (Markwardt \& Ögelman 1995). We present data obtained by the X-ray ASCA satellite which confirms the enhanced Xray emission at the jet head and discuss the allowable spectral models. The jet parameters are updated according to the combined ROSAT and ASCA results.
\end{abstract}

ROSAT observations of the Vela pulsar and its vicinity have revealed the existence of a one sided jet-like outflow from the pulsar (Markwardt \& Ögelman 1995). The jet, which is 45 arcmin long by 12 arcmin wide $(1.5 \mathrm{pc} \times 7 \mathrm{pc}$ at a distance of $500 \mathrm{pc}$ ), appears only in the $0.7-2.4 \mathrm{keV}$ energy band of ROSAT. In the lower energy channels the soft emission from the Vela supernova remnant (SNR) dominates and the jet is not visible. With the limited ROSAT spectral resolution $(\Delta E / E \sim 50 \%$ at $1 \mathrm{keV})$ a unique spectral model for the jet emission could not be found.

In the narrow bandpass of $0.7-2.4 \mathrm{keV}$, the jet spectrum can be modeled by emission from either a thermal plasma or a power law continuum, but the powerlaw model was inconsistent with ROSAT data when extended to low energies. The observed ROSAT spectrum is best-fit by emission from a thermal plasma with a temperature of $1.3 \mathrm{keV}$ and density of $0.4 \mathrm{~cm}^{-3}$. Because the emission is centrally bright, the jet feature was interpreted as a filled "cocoon" of heated gas, produced by the jet material shocking against the ambient SNR. A simple momentum- and mass-conserving model of the jet and its cocoon allowed an estimate of the important jet parameters, listed in Table 1. The derived mechanical energy of the jet was about $50 \%$ of the pulsar's spindown luminosity of $7 \times 10^{36} \mathrm{erg} \mathrm{s}^{-1}$, which further confirms its interpretation as a pulsar jet.

Table 1. The parameters of the Vela Jet. The jet speed is $v_{j}$ and the mass flow rate is $\dot{M}$.

\begin{tabular}{lll}
\hline Parameter & ROSAT & ASCA + ROSAT \\
\hline$v_{j}$ & $1100 \mathrm{~km} / \mathrm{s}$ & $\geq 950 \mathrm{~km} / \mathrm{s}$ \\
$\dot{M}$ & $10^{-5} M_{\odot} / \mathrm{yr}^{-1}$ & $\geq 2 \times 10^{-6} M_{\odot} / \mathrm{yr}$ \\
Power & $4 \times 10^{36} \mathrm{erg} \mathrm{s}^{-1}$ & $10^{36}-10^{37} \mathrm{erg} \mathrm{s}^{-1}$ \\
\hline
\end{tabular}



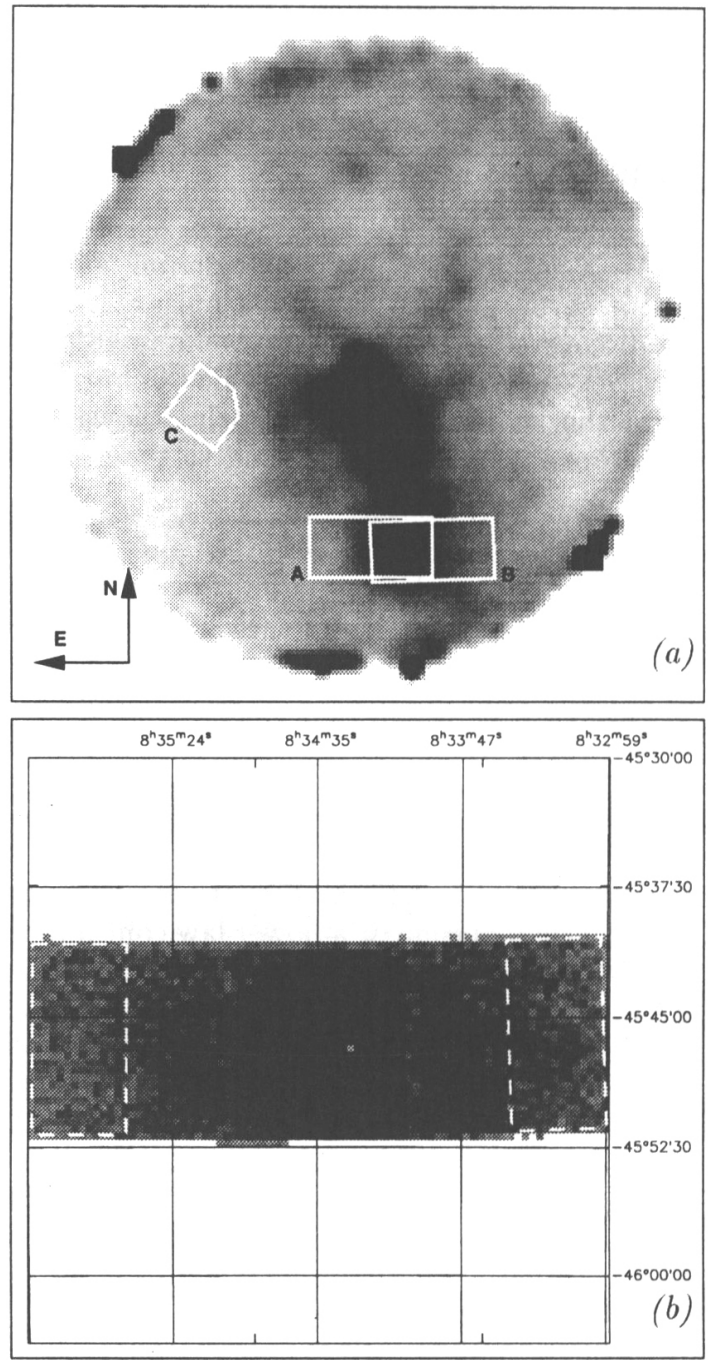

Figure 1. (a) ROSAT X-ray image $\left(2^{\circ} \times 2^{\circ}\right)$ from $0.7-2.4 \mathrm{keV}$ showing the X-ray jet from the pulsar and the observed ASCA fields (each $11 \times 22 \operatorname{arcmin}^{2}$ ), identified as pointings A \& B. Pointing C was an archival observation far from the jet used to verify that the background was uniform. (b) ASCA image corresponding to combined pointings A $\&$ B showing the enhanced jet emission at the center. Selection regions outlined in white were used for background subtraction. 
Table 2. Fitted jet and SNR head spectral parameters.

\begin{tabular}{|c|c|c|c|}
\hline \multicolumn{4}{|c|}{ Raymond \& Smith (Soft+Hard) } \\
\hline Comp. & Temp.(keV) & Frac.of flux & $n_{\varepsilon}\left(\mathrm{cm}^{-3}\right)$ \\
\hline Soft & 0.3 & $50 \%$ & 0.41 \\
\hline Hard & 3.7 & $50 \%$ & 0.33 \\
\hline \multicolumn{4}{|c|}{ also require abundances of $[\mathrm{O}]=0.3$ and $[\mathrm{Fe}]=0.1$} \\
\hline \multicolumn{4}{|c|}{ Raymond \& Smith + Power Law } \\
\hline Comp. & Temp.(keV) & Frac.of flux & $n_{e}\left(\mathrm{~cm}^{-3}\right)$ \\
\hline$\overline{\mathrm{RS}}$ & 0.3 & $35 \%$ & 0.30 \\
\hline PL & $\alpha=-2.1$ & $65 \%$ & - \\
\hline \multicolumn{4}{|c|}{ also require abundances $[\mathrm{Ne}]=1.6,[\mathrm{O}]=0.5,[\mathrm{Fe}]=0.2$} \\
\hline \multicolumn{4}{|c|}{ SNR with 3 component Raymond \& Smith model } \\
\hline Comp. & & Temp.(keV) & $n_{e}\left(\mathrm{~cm}^{-3}\right)$ \\
\hline Softest ( & uter shell) & 0.1 & - \\
\hline Soft (she & & 0.3 & - \\
\hline Hard (in & ide $\mathrm{R}=50 \mathrm{pc}$ ) & 4.5 & 0.06 \\
\hline
\end{tabular}

In order to gain insight on the emission mechanism of the jet we have performed additional observations of the head of the jet with the ASCA Xray telescope. The ASCA SIS instrument is sensitive to higher energy $\mathrm{X}$-rays, $0.5-10 \mathrm{keV}$, and has spectral resolution of $\sim 5 \%$ at $1 \mathrm{keV}$, much better than ROSAT. Figure 1a shows the observed ASCA SIS fields of view superimposed on the ROSAT image. Figure $1 \mathrm{~b}$ shows the corresponding ASCA image obtained of the jet head. The enhancement in $\mathrm{X}$-ray emission from the jet is $2-3 \times 10^{-3}$ ct s $^{-1} \operatorname{arcmin}^{-2}$, and is clearly seen in the image.

The spectrum of the jet was obtained after subtraction of the SNR background selected from the indicated regions of Figure $1 \mathrm{~b}$. Figure 2 shows the combined ROSAT+ASCA spectrum of both the SNR and the Head+SNR, which extend to energies of $\sim 7 \mathrm{keV}$ (the sensitivity limit). The head spectrum is remarkably smooth except for the spectral peak near $0.9 \mathrm{keV}$, suggestive of Neon IX emission from gas below temperatures of $0.3 \mathrm{keV}$. The high energy portion of the spectrum however, demands temperatures of $\sim 4 \mathrm{keV}$ if thermal. Because a single emission model is clearly not consistent with the data, twocomponent models consisting of a soft Raymond \& Smith (1977) thermal component and a harder thermal/power-law component were fitted to the combined ROSAT+ASCA spectrum and the results are shown in Table 2.

A power law emission model is consistent with the observed $0.1-7.0 \mathrm{keV}$ energy range only if a second thermal component is added. Thus, even if the higher energy part of the spectrum is interpreted as synchrotron emission there is still a thermal component of the jet peaked near $\sim 1 \mathrm{keV}$. Using the fitted parameters of the thermal model obtained from ASCA we have revised the estimated jet properties and taken into account the uncertainty in the angle of inclination. The comparative results are shown in Table 1. 


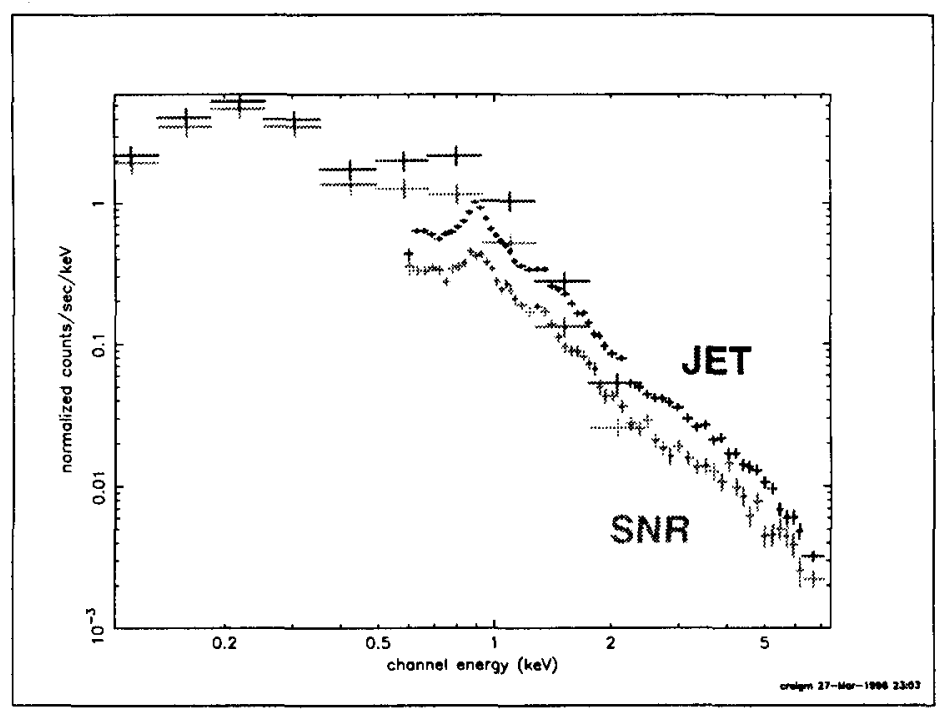

Figure 2. Combined ROSAT and ASCA spectrum of the Vela jet head and the surrounding SNR in units of counts $\mathrm{s}^{-1} \mathrm{keV}^{-1}$ per unit $\mathrm{CCD}$ area of $11 \times 11 \mathrm{arcmin}^{2}$. ROSAT bins are in the range 0.1$2.4 \mathrm{keV}$ and are wide, while ASCA bins are narrow and in the range $0.7-7.0 \mathrm{keV}$. The Head+SNR spectrum (dark points) is about twice as intense as the SNR alone (light points) but has almost the same shape above $0.7 \mathrm{keV}$. Below $0.7 \mathrm{keV}$ the emission from the SNR dominates and the two spectra are nearly indistinguishable.

The primary surprises from the new ASCA results are that the jet and SNR spectra are very similar (differing only in intensity above $0.7 \mathrm{keV}$ ) and that both the jet and SNR spectrum are lacking emission line features, unless the lines are so severely blended that they form a continuum. The shocks must be weak at the head of the jet since there is no significant temperature difference between the cocoon and the SNR. Still, the energy flow in the jet remains comparable to the spindown luminosity of the pulsar, and indicates that it is connected to an outflow along the rotation axis of the pulsar. With the observation of jetlike signatures associated with this and other pulsars, we suggest that jets may be more ubiquitous than previously imagined and that a search for jets around other pulsars may prove fruitful.

\section{References}

Raymond, J.C. \& Smith, B.W. 1977, ApJS, 35, 419.

Markwardt, C.B. \& Ögelman, H. 1995, Nature, 375, 40. 